\title{
Estudio empírico en plantas de beneficio de carbón mineral localizadas en San Javier, Sonora, México, para diseñar un sistema sostenible de producción más limpia *
}

\author{
Empirical Study in Mineral Coal Beneficiation Plants Located in San Javier, Sonora, Mexico, to Design a Sustainable \\ Cleaner Production System \\ Estudo empírico em plantas de beneficiamento de carvão mineral localizadas em San Javier, Sonora, México, para \\ projetar um sistema sustentável de produção mais limpa
}

Luis Enrique Duarte-Verdugo a

Universidad de Sonora, México

leduarte@prodigy.net.mx

ORCID: http://orcid.org/0000-0001-9483-6797

DOI: https://doi.org/10.11144/Javeriana.cc18-45.eepb

Juan Carlos Robles-Ibarra

Universidad de Sonora, México

ORCID: http://orcid.org/0000-0003-3401-3574

Beatriz Pico-González

Universidad Popular Autónoma del Estado de Puebla,

México

ORCID: http://orcid.org/0000-0002-7619-0336

Genoveva Rosano-Ortega

Universidad Popular Autónoma del Estado de Puebla,

México

ORCID: http://orcid.org/0000-0002-7297-3456

\section{Resumen:}

En esta investigación se realizó un estudio empírico en plantas de beneficio de carbón mineral ubicadas en el municipio de San Javier, en el estado de Sonora, en México, con el objetivo de diseñar un modelo sostenible de producción más limpia que les dé solución a los problemas que generan estas plantas de beneficio de carbón mineral, cuando están en operación. Se identificó como problema principal que las plantas de beneficio de carbón no contribuyen al desarrollo sostenible, pues generan una gran cantidad de emisiones, que afectan el recurso humano que labora en estas plantas e impactan negativamente en el medio ambiente que las rodea. Para obtener la información requerida en el diseño del modelo y el logro de los objetivos, se utilizaron dos instrumentos: una encuesta y la Matriz de Leopold. Con ambos instrumentos se obtuvieron resultados que sustentaron el modelo propuesto. El modelo obtenido en esta investigación es de aplicación general, ya que se puede utilizar en cualquier sistema de producción.

Código JEL: Q01

Palabras clave: Estudio empírico, producción más limpia, desarrollo sostenible, medio ambiente.

\begin{abstract}
:
In this research an empirical study was carried out in coal beneficiation plants located in the municipality of San Javier, in the state of Sonora, in Mexico, aiming to design a sustainable model of cleaner production that solves the problems generated by these mineral coal beneficiation plants, when they are in operation. The main problem identified was that coal beneficiation plants do not contribute to sustainable development, because they generate a large amount of emissions, which affect the human resources that work in these plants and negatively impact the surrounding environment. Two instruments were used to obtain the information required in the design of the model and the achievement of the objectives: a survey and the Leopold Matrix. With both instruments, results were obtained that supported the proposed model. The model obtained in this research is of general application, given that it can be used in any production system.
\end{abstract}

Notas de autor 
Keywords: Empirical study, cleaner production, sustainable development, environment.

Resumo:

Nesta pesquisa realizou-se um estudo empírico em plantas de beneficiamento de carvão mineral localizadas no município de San Javier, no estado de Sonora, no México, a fim de projetar um modelo sustentável de produção mais limpa que dê solução aos problemas que geram estas plantas de beneficiamento de carvão mineral, quando em operação. Identificou-se como problema principal que as plantas de beneficiamento de carvão não contribuem ao desenvolvimento sustentável, pois geram grande quantidade de emissões que afetam o recurso humano que labora nestas plantas e impactam negativamente no meio ambiente que as rodeia. Para obter a informação requerida no design do modelo e a consecução de objetivos, utilizaram-se dois instrumentos: um inquérito e a Matriz de Leopold. Com ambos os instrumentos obtiveram-se resultados que sustentaram o modelo proposto. $\mathrm{O}$ modelo obtido nesta pesquisa é de aplicação geral, pois pode se utilizar em qualquer sistema de produção.

Palavras-chave: Estudo empírico, produção mais limpa, desenvolvimento sustentável, meio ambiente.

\section{Introducción}

En la actualidad, los mayores conocimientos geológicos y económicos de las zonas carboníferas de México se tienen en las subcuencas del distrito Monclova-Sabinas en el estado de Coahuila, las subcuencas en el distrito Tezoatlán-Mixtepec en el estado de Oaxaca y las de San Javier-San Marcial en la cuenca de Barrancas en el estado de Sonora (Flores-Galicia, 1988).

En las subcuencas de San Javier-San Marcial en el estado de Sonora hay cinco plantas de beneficio de carbón mineral en operación, en las cuales se realizó un estudio empírico. En esta zona, ya se han realizado investigaciones, pero únicamente sobre los yacimientos de carbón mineral; sin embargo, no se encontraron investigaciones que se puedan tomar como referencia para conocer las condiciones de operación de las plantas de beneficio de este mineral y su contribución al desarrollo sostenible mediante sistemas de producción más limpia.

El común denominador en las cinco plantas cuando están en operación es su gran cantidad de emisiones (figura 1), debido a que no cuentan con tecnologías limpias, lo cual afecta las condiciones de trabajo en las que el personal que labora en estas plantas desempeña sus funciones, además de impactar en los factores tanto bióticos como abióticos de las inmediaciones de estas plantas. Lo anterior trae como consecuencia que no se respeten las políticas ambientales.

Los inconvenientes antes mencionados se pueden considerar como antecedentes para determinar que esta actividad económica no contribuye al desarrollo sostenible, como lo establecen los siguientes autores citados en esta investigación.

Gavin Hilson y Barbara Murck (2000) afirman que para que una empresa minera contribuya al desarrollo sostenible debe implementar sólidas herramientas de gestión ambiental y utilizar tecnologías limpias.

Béatrice Labonne (1999) considera que un requisito para que una empresa minera contribuya al desarrollo sostenible, debe minimizar el impacto ambiental en todas las etapas del ciclo de vida del proyecto: exploración, explotación y clausura del proyecto.

Para Roderick G. Eggert (2000), una empresa minera puede contribuir al desarrollo sostenible al mantener y mejorar las condiciones de vida de la comunidad, mediante la creación de empleos bien remunerados y con condiciones seguras de trabajo para sus residentes.

Según Tim Jackson (1993), en el cuidado del medio ambiente se tienen dos opciones: la primera se refiere a la prevención y la segunda es la reacción.

La reacción se relaciona con el control de la contaminación, es decir, son las acciones que se ejecutan una vez que los efectos de la contaminación se han presentado, en contraparte la prevención son las medidas adoptadas para influir en las posibles causas de los efectos adversos. 


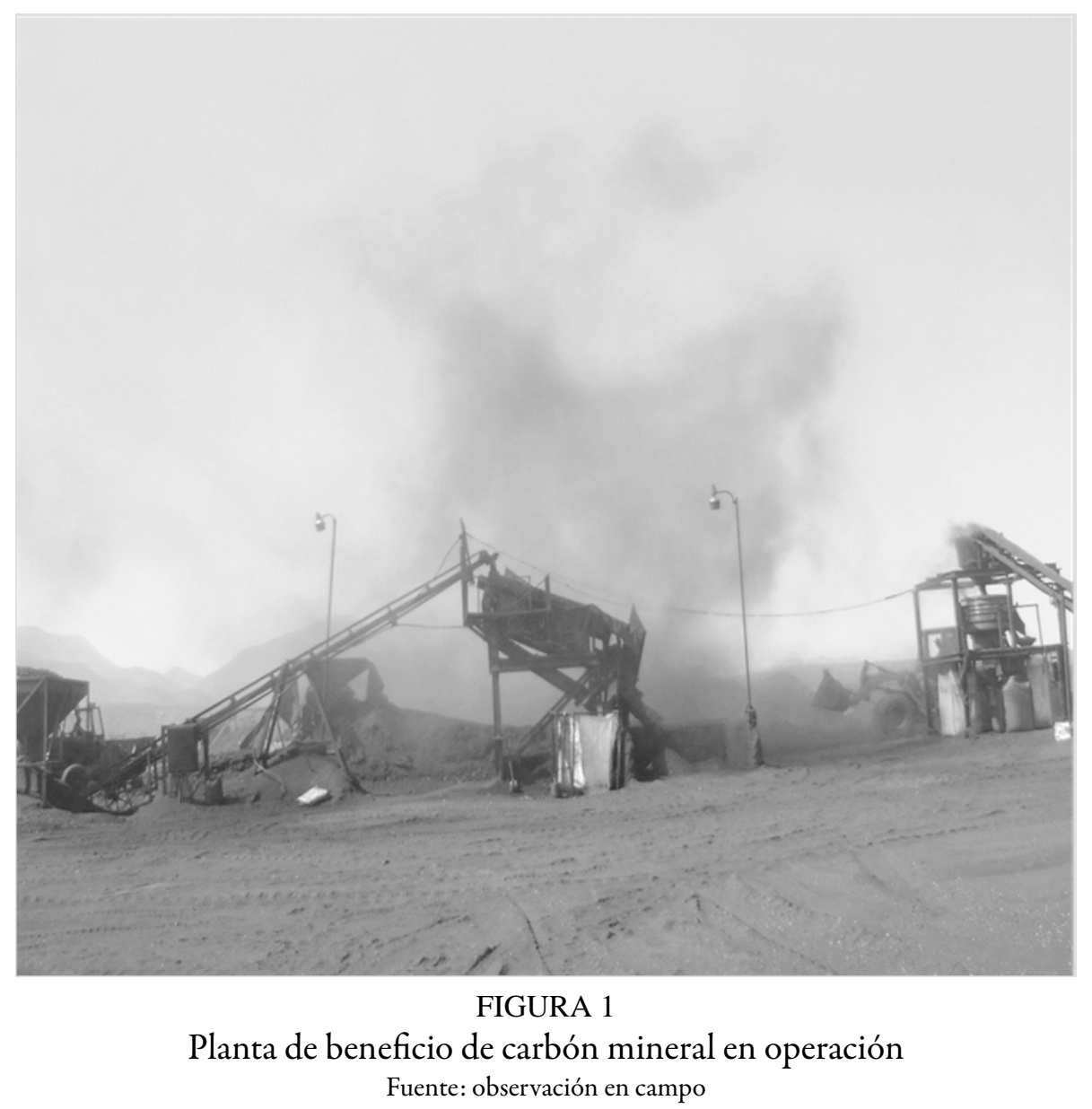

El propósito de esta investigación es diseñar una propuesta que se pueda considerar como una alternativa que solucione los problemas generados por las condiciones en que trabajan las cinco plantas de beneficio de carbón mineral en el municipio de San Javier en el estado de Sonora.

Para la justificación de esta investigación se tomó como referencia el Principio I de la Declaración de Río sobre el Medio Ambiente y el Desarrollo (ONU, 1992) que establece: "Los seres humanos constituyen el centro de las preocupaciones relacionadas con el desarrollo sostenible y tienen derecho a una vida saludable y productiva en armonía con la naturaleza”.

Es imposible lograr el principio antes mencionado sin una adecuada tecnología, como lo establece la Resolución A/RES/64/212 (ONU, 2010), aprobada por la Asamblea General de la Organización de las Naciones Unidas el 21 de diciembre de 2009, que reconoce la función decisiva que tienen la ciencia y tecnología, incluso las tecnologías ecológicamente racionales, en la protección del medio ambiente, así como en el mejoramiento de la productividad y competitividad (ONU, 2010).

El objetivo consistió en diseñar un modelo sostenible de producción más limpia, mediante un estudio empírico realizado en las plantas de beneficio de carbón mineral ubicadas en el municipio de San Javier, Sonora, y los objetivos específicos son los siguientes:

- Conocer los elementos que integran el modelo sostenible de producción más limpia.

- Identificar las estrategias que se integran en función de la misión, visión y el análisis de fortalezas, oportunidades, debilidades, amenazas, FODA, para el desarrollo de un modelo sostenible de producción más limpia.

- Conocer las políticas que integran el diseño de un modelo sostenible de producción más limpia. 
- Identificar los elementos que integran el diagnóstico para el diseño de un modelo sostenible de producción más limpia.

Como ya se hizo mención, el estudio se realizó en el municipio de San Javier, Sonora, ubicado en el centro del estado de Sonora, a 147 kilómetros de Hermosillo, la capital de Sonora. Su cabecera es la población de San Javier y se localiza en el paralelo $28^{\circ} 36^{\prime}$ de latitud norte y a los $109^{\circ} 44^{\prime}$ de longitud al oeste del meridiano de Greenwich, a una altura de 650 metros sobre el nivel del mar. Colinda con los municipios de Soyopa al norte, Onavas al este, Suaqui Grande al sur y la Colorada al oeste. Las actividades principales en San Javier son la ganadería y la minería. La figura 2 muestra la población en los cuatro censos más recientes.

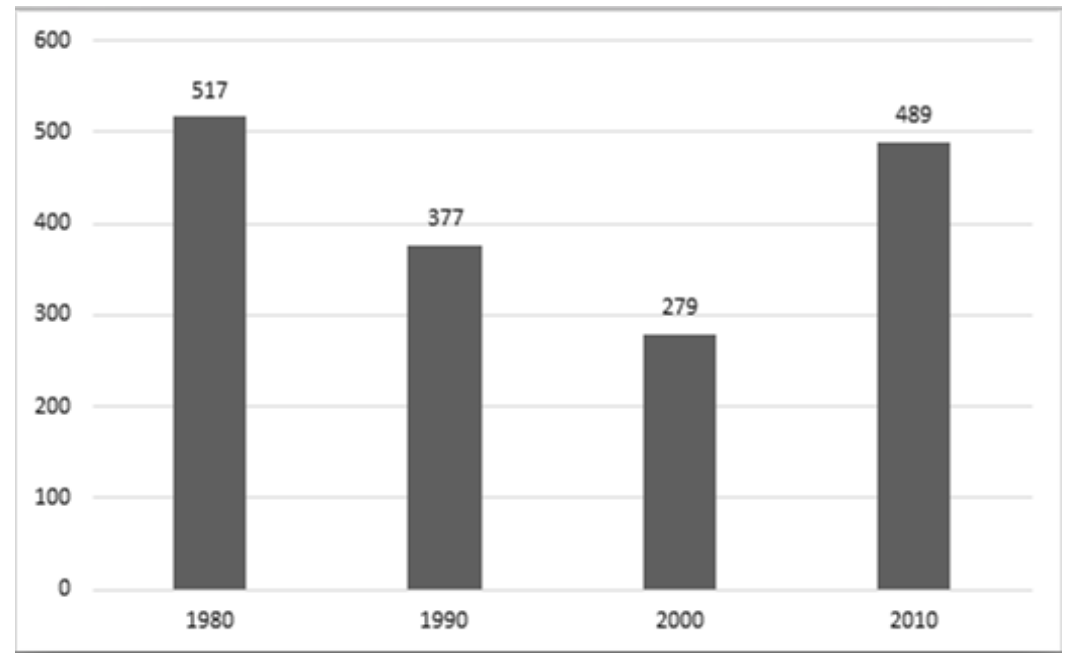

FIGURA 2

Población en San Javier, Sonora (1980-2010)

Fuente: Instituto Nacional de Estadística y Geografía, INEGI

Hay 5 plantas de beneficio de carbón mineral instaladas en esta región, que generan unos 250 empleos. En la figura 3 se observa el volumen histórico en toneladas de carbón antracítico extraídas en el estado de Sonora en el período 2006-2014 (Secretaría de Economía del Estado de Sonora, 2014).

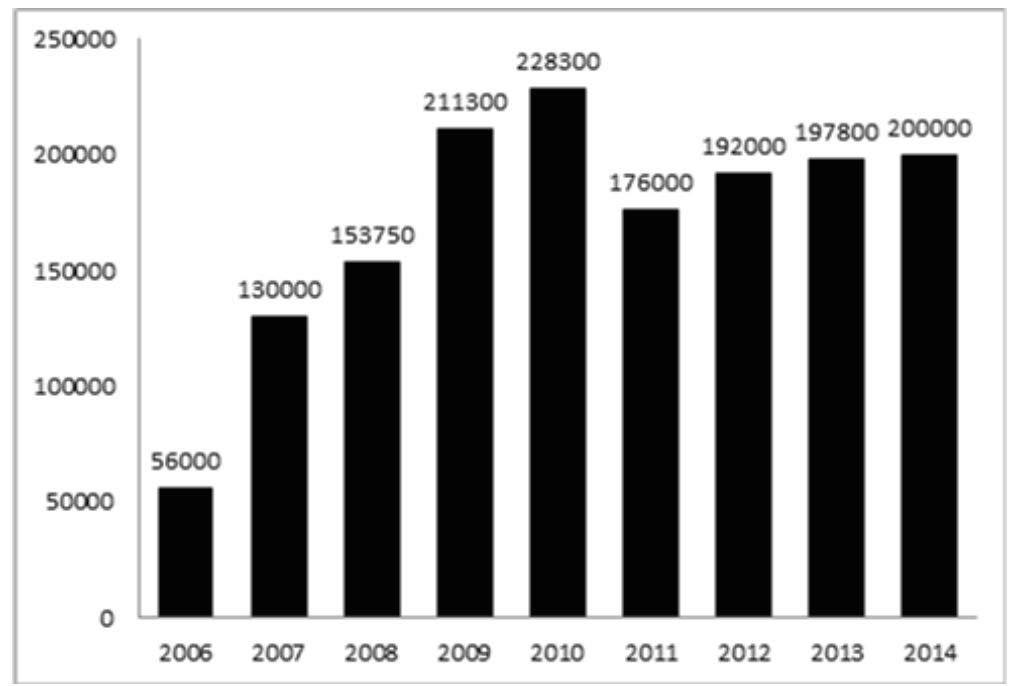

FIGURA 3

Volumen histórico de producción de carbón antracítico en Sonora (toneladas)

Fuente: Secretaría de Economía del Estado de Sonora (2014) 


\section{Metodología de la investigación}

Para obtener la información que se requiere en el diseño del modelo sostenible de producción más limpia propuesto, se hizo un estudio empírico en las plantas de beneficio de carbón mineral ubicadas en San Javier, Sonora.

Renate Mayntz, Kurt Holm y Peter Hübner (1988) definen el estudio empírico como la investigación basada en la observación para descubrir algo desconocido o probar una hipótesis.

El enfoque de esta investigación es mixto: cuantitativo y cualitativo. Cuantitativo, porque se recolectaron datos para probar hipótesis, y cualitativo, porque se utilizó la recolección de datos sin medición numérica para descubrir o afinar preguntas de investigación en el proceso de interpretación (Hernández-Sampieri, Fernández-Collado \& Baptista-Lucio, 2010).

Los enfoques tanto cuantitativos como cualitativos, según Richard M. Grinnell e Yvonne Unrau (1977), emplean procesos tanto metódicos como empíricos en sus esfuerzos para generar conocimiento.

Además del enfoque antes mencionado, el alcance de esta investigación es exploratorio, descriptivo, correlacional y explicativo.

Tomando como referencia el concepto de población de Claire Selltiz, Lawrence Wrightsman y Stuart W. Cook (1980), como el conjunto de todos los casos que concuerdan con una serie de especificaciones, el objeto de estudio en esta investigación lo representan las plantas de beneficio de carbón mineral que se ubican en el municipio de San Javier, en el estado de Sonora, y que están en operación actualmente.

Dado que la población es pequeña, se decidió trabajar con el total de la población que está representada por las 5 plantas de beneficio de carbón mineral ubicadas en San Javier, Sonora.

Para la selección y elaboración de los instrumentos se consideró lo expuesto por Richard M. Grinnell, Margaret Williams e Yvonne Unrau (2009), quienes establecen que un instrumento debe registrar únicamente datos observables y que representen verdaderamente los conceptos o las variables que el investigador tiene en mente. Para tal efecto, esta investigación utilizó como instrumentos la encuesta y la Matriz de Leopold:

- La encuesta se estructuró con preguntas que se obtuvieron de las listas de verificación del aire, el impacto ambiental y el suelo, que utiliza la Procuraduría Federal de Protección al Ambiente (PROFEPA) en sus auditorías ambientales a todo tipo de industrias, debido a que no se puede entender un sistema de producción más limpia sin la seguridad e higiene de los trabajadores que laboran en estas plantas. Se incluyeron en la encuesta algunas preguntas sobre Normas Oficiales Mexicanas, NOM, que se deben cumplir para garantizar la seguridad de los trabajadores en los centros de trabajo.

- Matriz de Leopold. Esta fue diseñada inicialmente para evaluar los impactos ambientales que se relacionaban con proyectos mineros (Leopold, Clarke, Hanshaw \& Balsley, 1971). En las filas se presentan los factores ambientales que pueden ser afectados y en las columnas, las acciones que se realizarán y que pueden causar impacto en los factores. En la tabla 1 se observa la matriz utilizada en esta investigación. 
TABLA 1

Matriz de Leopold

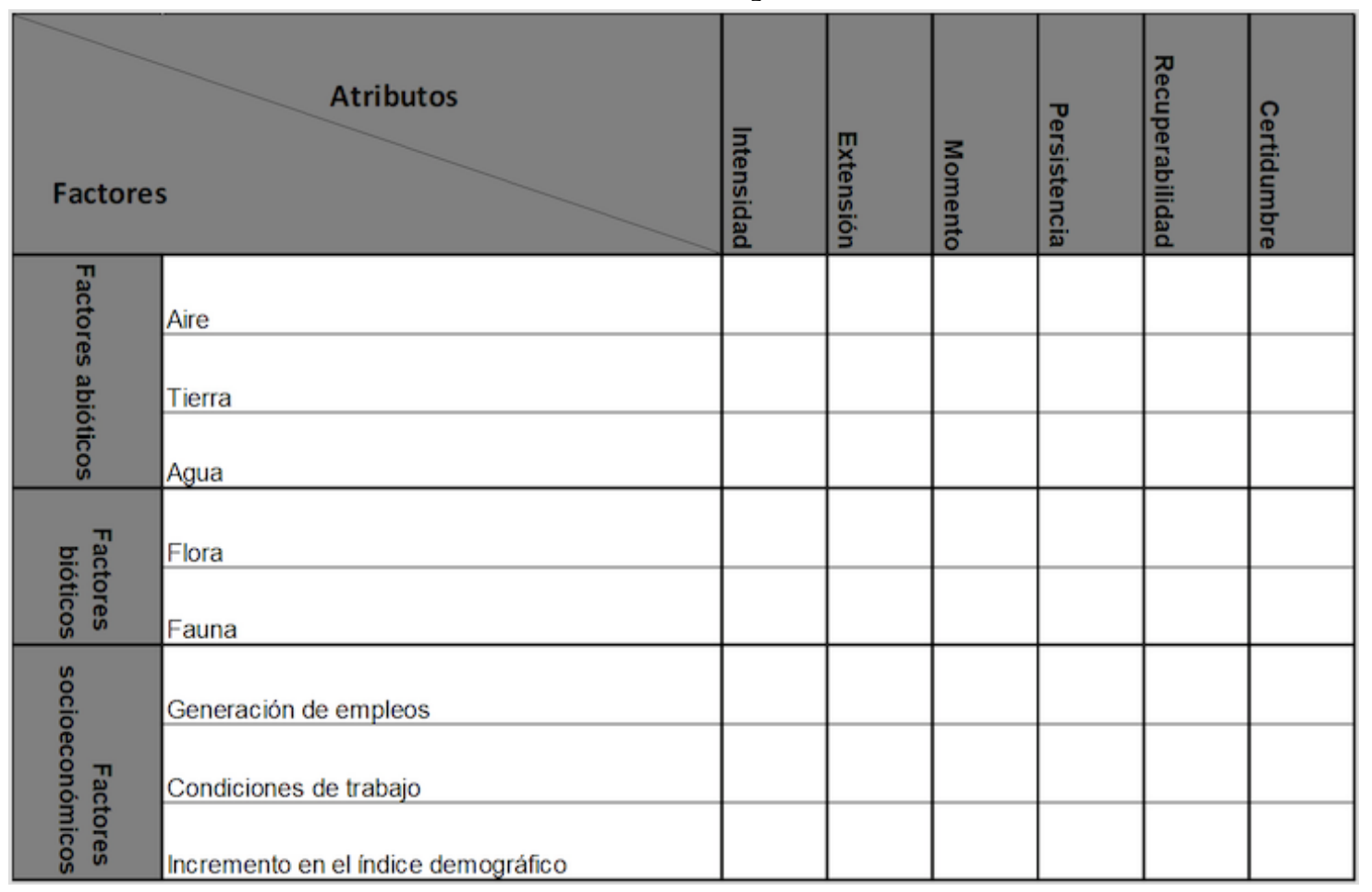

Fuente: elaboración propia, con base en Ignacio Daniel Coria (2008)

El método Delphi se utilizó para obtener la información que se requiere en la estructura de la matriz de Leopold; por medio de una carta formal enviada vía correo electrónico, se solicitó a un grupo de cuatro expertos con más de 30 años de experiencia en actividades mineras su participación y colaboración en esta investigación, se les explicó claramente en qué consiste y qué objetivos se pretenden.

Eduardo Kazuo Kayo y Jose Roberto Securato (1997) señalan que el método Delphi puede tener innumerables variaciones, pero el método convencional se caracteriza por ser un cuestionario enviado a los especialistas en espera de su retorno; en este caso, el principal inconveniente es la demora de las respuestas.

Según Martínez (2005), para el desarrollo del método Delphi se deben considerar las cuatro fases que se presentan en la tabla 2. 
TABLA 2

Fases para la implementar el Método Delphi

\begin{tabular}{|c|c|}
\hline FASE & DESCRIPCIÓN \\
\hline 1.- Formulación del problema. & $\begin{array}{l}\text { Conocer el impacto ambiental que generan } \\
\text { las plantas de beneficio de carbón mineral } \\
\text { ubicadas en la zona centro del estado de } \\
\text { Sonora. }\end{array}$ \\
\hline $\begin{array}{l}\text { 3.- Elaboración y lanzamiento del } \\
\text { cuestionario. }\end{array}$ & $\begin{array}{l}\text { Considerando lo establecido por (Ludwig, } \\
\text { 1994) se determinó trabajar con } 4 \text { expertos en } \\
\text { el tema del impacto ambiental que causan las } \\
\text { plantas de beneficio de carbón mineral } \\
\text { cuando estas están en operación, distribuidos } \\
\text { de la siguiente manera: } \\
\text { 1.- Funcionario de fomento minero del } \\
\text { G obierno del estado de Sonora. } \\
\text { 2.- Investigadores de la Universidad de } \\
\text { Sonora } \\
\text { 1.- Empresario minero. } \\
\text { Se les envió a los expertos una carta en donde } \\
\text { se explica en que consiste el proyecto y la } \\
\text { manera en que deben elaborar la matriz de } \\
\text { Leopold basándose en la información } \\
\text { proporcionada delos atributos a evaluar. }\end{array}$ \\
\hline $\begin{array}{l}\text { 4.- Desarrollo práctico y } \\
\text { explotación de resultados. }\end{array}$ & $\begin{array}{l}\text { En el capitulo } 5 \text { de resultados y analisis se } \\
\text { presentan los resultados obtenidos. }\end{array}$ \\
\hline
\end{tabular}

Fuente: elaboración propia, con base en Martínez (2005)

\section{Modelo sustentable de producción más limpia propuesto}

El Programa de las Naciones Unidas para el Medio Ambiente, PNUMA, define la producción más limpia como "la aplicación continua de una estrategia ambiental preventiva integrada a los procesos, a los productos y a los servicios, para incrementar la eficiencia y reducir los riesgos relevantes de los seres humanos y el medio ambiente" (Espinosa-Murga, 2007).

Tim Jackson (1993) define la producción más limpia como "Un enfoque operativo para el desarrollo de los sistemas de producción y de consumo que incorpora la prevención en la protección del medio ambiente”.

En la integración de la investigación tanto documental como de campo, surgieron tres elementos indispensables para desarrollar el modelo sostenible de producción más limpia: diagnóstico, políticas y estrategia. En la figura 4 se puede observar el modelo propuesto con estos elementos. 


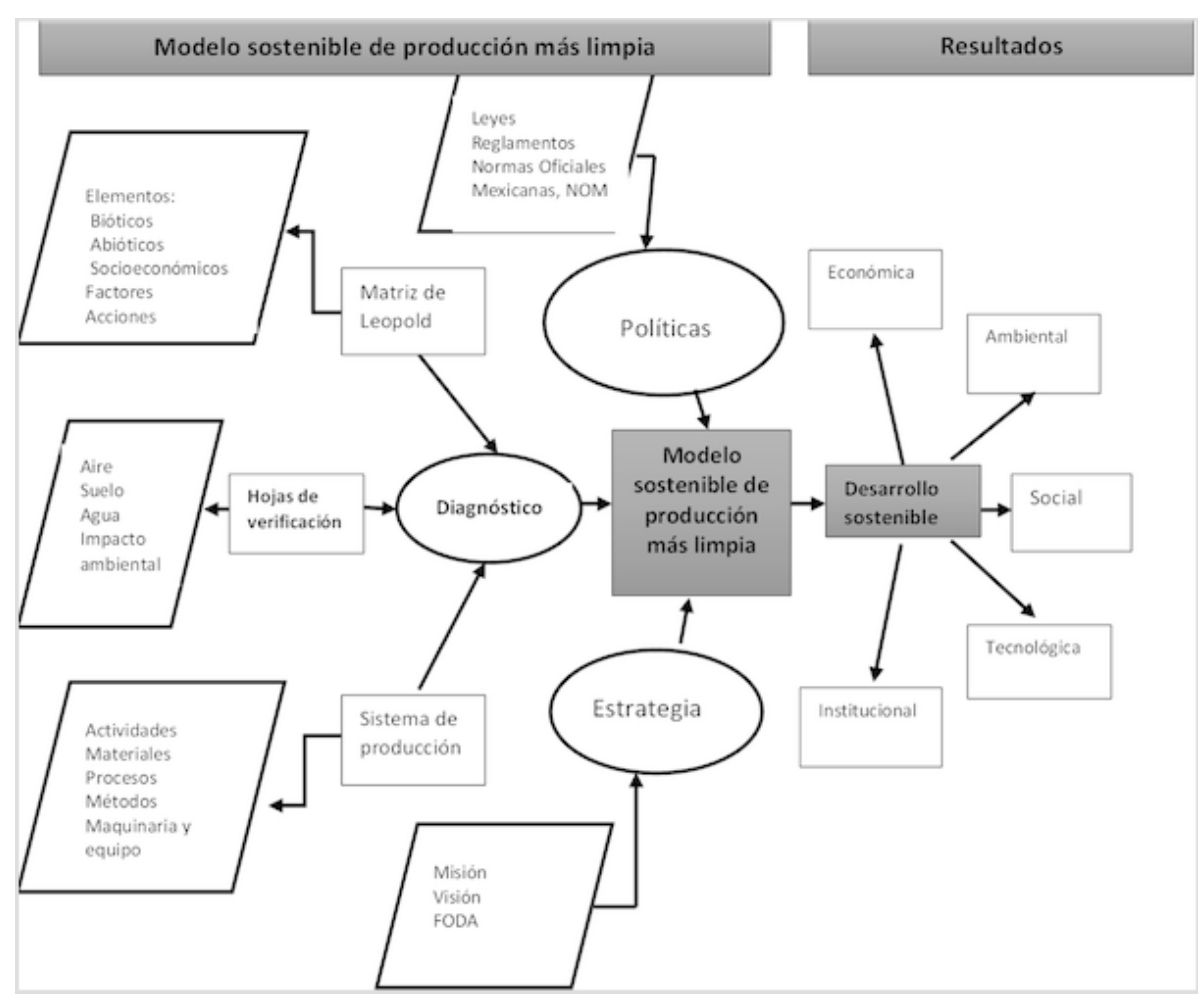

FIGURA 4

Modelo sostenible de producción más limpia propuesto

Fuente: elaboración propia

\section{Resultados}

La información requerida para generar una propuesta de solución al problema antes planteado y el logro de los objetivos establecidos, se obtuvo mediante los dos instrumentos antes mencionados.

Primero se presentarán los resultados obtenidos en la encuestas y luego los resultados que se obtuvieron en la Matriz de Leopold.

\section{Resultados de la encuesta}

Las preguntas aplicadas están basadas en las listas de verificación del aire, suelo e impacto ambiental que utiliza la PROFEPA en sus auditorías ambientales; además se incluyeron preguntas sobre Normas Oficiales Mexicanas (NOM) que se deben cumplir para garantizar condiciones seguras de trabajo.

La tabla 3 presenta los resultados de las plantas que cuentan con los documentos que se requieren para obtener el permiso de operación.

TABLA 3

Verificación de documentos

\begin{tabular}{|l|c|c|}
\hline \multicolumn{1}{|c|}{ Descripción } & Sí & No \\
\hline ¿Cuenta la empresa con licencia ambiental única? (Art. 18 RLGEEPAATM) & 5 & 0 \\
\hline ¿Cuenta la empresa con licencia de uso del suelo? (Art. 23 LGEEPA) & 5 & 0 \\
\hline ¿La instalación cuenta con autorización en materia de impacto ambiental? (Art. 28 LGEEPA) & 5 & 0 \\
\hline
\end{tabular}




\section{Fuente: elaboración propia}

En la tabla 3 se puede observar que las cinco plantas encuestadas tienen toda documentación en regla.

En la tabla 4 se presentan los resultados obtenidos de las preguntas que están incluidas en la Guía de Verificación del Aire proporcionada por la PROFEPA delegación Sonora.

TABLA 4

Guía de verificación del aire

\begin{tabular}{|c|c|c|c|}
\hline Preguntas de la encuesta & Descripción & Si & No \\
\hline 7 & ¿En las instalaciones se generan emisiones a la atmós fera? (Art. 10 RLGEEPAATM) & 5 & 0 \\
\hline 8 & ¿Las emisiones están conducidas? (Art. 23 RLGEEPAATM) & 0 & 5 \\
\hline 9 & Se cuenta con equipos colectores de polvos? (Art. 17 RLGEEPAATM) & 0 & 5 \\
\hline 10 & ¿Se detecta contaminación de la atmósfera en áreas vecinas? (Art.110 LGEEPA) & 5 & 0 \\
\hline 11 & Se tienen en las instalaciones aspersores para aplicación de agua? (Art. 17 RLGEEPAATM) & 0 & 5 \\
\hline 12 & ¿Se tienen en las instalaciones cortinas rompe vientos? (Art. 17 RLGEEPAATM) & 0 & 5 \\
\hline 13 & Se cuenta con un programa de mantenimiento de los equipos? (BOPI ART. 4 RLGEEPAAA) & 5 & 0 \\
\hline
\end{tabular}

Fuente: elaboración propia

La tabla 4 muestra resultados muy contundentes: todas las empresas encuestadas impactan negativamente en el aire. Las respuestas de las preguntas así lo demuestran; sin embargo, ninguna planta cuenta con tecnologías limpias que pudieran mitigar este impacto.

La tabla 5 presenta los resultados obtenidos de las preguntas que están incluidas en la Guía de Verificación del suelo.

TABLA 5

Guía de verificación del suelo

\begin{tabular}{|c|l|c|c|}
\hline Preguntas de encuesta & & Descripción & No \\
\hline 14 & ¿Hay derrames de materiales o residuos peligrosos en el suelo? (Art. 70 LGPGIR) & \\
\hline 15 & ¿En las vecindades hay suelos contaminados? (Art. 70 LGPGIR) & 0 \\
\hline 16 & Si la respuesta anterior fue si, ¿se han hecho estudios de evaluación y acciones de restauración? (Art. 69 LGPGIR) & 0 & 0 \\
\hline 17 & ¿Se tienen en operación tanques o diques de almacenamiento? & 0 & 5 \\
\hline 18 & Si la respuesta anterior fue sí, ¿se cuenta con estructuras de control de derrames? (BOPI, Art. 4RLGEEPAAA) & 0 & 0 \\
\hline
\end{tabular}

Fuente: elaboración propia

Debido a la naturaleza del proceso de producción, el cual consiste solo en el cambio físico del mineral de carbón, no se utilizan productos químicos por lo tanto no hay derrames de materiales o residuos peligrosos; sin embargo, sí hay contaminación en suelos vecinos por la emisión de materiales finos que de alguna manera se depositan en estos; en este punto, la respuesta es que no se han hecho estudios de evaluación ni se han realizado acciones de restauración; así mismo, ninguna de las 5 plantas tiene en operación tanques de almacenamiento.

En la tabla 6 se muestran los resultados de las preguntas incluidas en la guía de verificación de impacto ambiental. Estas preguntas están estructuradas en tres apartados: flora, fauna y agua.

TABLA 6

Guía de verificación de impacto ambiental

\begin{tabular}{|l|c|c|}
\hline \multicolumn{1}{|c|}{ Pregunta } & Sí & No \\
\hline ¿Afecta la flora vecina? & 5 & 0 \\
\hline ¿Afecta la fauna? & 5 & 0 \\
\hline ¿Afecta mantos acuíferos? & 0 & 5 \\
\hline
\end{tabular}

Fuente: elaboración propia 
Los resultados observados en la tabla 6 indican que el total de las plantas encuestadas impactan en la flora y la fauna vecina a las plantas de beneficio de carbón mineral. Sin embargo, el impacto ambiental no tiene problemas en los mantos acuíferos, ya que ninguna de las 5 plantas afecta los mantos acuíferos.

La tabla 7 contiene los resultados de las preguntas que se relacionan con Normas oficiales que deben cumplir todo tipo de instalaciones para garantizar condiciones seguras de trabajo al recurso humano de la empresa. Estas preguntas se relacionan con las Normas Oficiales Mexicanas NOM-001-STPS-2008, NOM-004-STPS-1999 y NOM-017-STPS-2008, de la Secretaría del Trabajo y Previsión Social.

TABLA 7

Condiciones de trabajo

\begin{tabular}{|c|l|c|c|}
\hline Pregunta de la encuesta & \multicolumn{1}{|c|}{ Descripción } & Si & No \\
\hline 20 & ¿Hay condiciones seguras en el centro de trabajo? NOM-001-STPS-2008 & 5 \\
\hline 21 & ¿Se cuenta con sistemas de protección en maquinaria y equipo? NOM-004-STPS-1999 & 5 & 0 \\
\hline 22 & ¿Se le proporciona al personal equipo de seguridad? NOM-017-STPS-2008? & 5 & 0 \\
\hline
\end{tabular}

Fuente: elaboración propia

Independientemente de que al personal se le proporcione equipo de seguridad en las 5 plantas encuestadas, y de que haya sistemas de protección en maquinaria y equipo en las mismas 5 plantas, en la tabla 7 se puede observar que no hay condiciones seguras de trabajo en ninguna de las 5 plantas de beneficio. Esto se debe a la gran cantidad de materiales finos que se generan en la descarga, traslado y trituración del mineral de carbón dentro de las instalaciones.

\section{Resultados de la matriz de Leopold}

Los atributos evaluados en la matriz de Leopold por los expertos seleccionados y los valores que pueden tomar sus observaciones están en la tabla 8. Estos atributos fueron propuestos por Vicente Conesa Fernández-Vítora (2010). 
TABLA 8

Valores de los atributos

\begin{tabular}{|c|c|c|}
\hline Atributo & & Observaciones \\
\hline \multirow[b]{2}{*}{ Carácter } & Positivo & \\
\hline & Negativo & \\
\hline \multirow{3}{*}{$\begin{array}{c}\text { Intensidad (In) Representa la incidencia de la acción causal sobre el } \\
\text { factor impactado en el área en la que se produce el efecto }\end{array}$} & 1 & Afección mínima \\
\hline & 2 a 11 & Intermedia \\
\hline & 12 & Destrucción total \\
\hline \multirow{4}{*}{$\begin{array}{l}\text { Extensión (Ex) Área de influencia teórica del impacto en relación } \\
\text { con el entomo del provecto }\end{array}$} & 1 & Impacto puntual \\
\hline & 2 & Impacto parcial \\
\hline & 4 & Impacto extenso \\
\hline & 8 & Impacto total \\
\hline \multirow{4}{*}{$\begin{array}{c}\text { Momento (Mo) Se refiere al tiempo transcurrido entre la acción y la } \\
\text { aparición del impacto }\end{array}$} & 4 & Inmediato \\
\hline & 3 & Corto plazo (menos de un año) \\
\hline & 2 & Mediano plazo (de 1 a 5 años) \\
\hline & 1 & Largo plazo (más de 5 años) \\
\hline \multirow{3}{*}{$\begin{array}{c}\text { Persistencia (Pe) Se refiere al tiempo que el efecto se manifiesta } \\
\text { hasta que se retome a la situación inicial en forma natural o por } \\
\text { medio de medidas correctoras }\end{array}$} & 1 & Fugaz \\
\hline & 2 & Temporal ( de 1 a 10 años) \\
\hline & 4 & Permanente (más de 10 años) \\
\hline \multirow{3}{*}{$\begin{array}{l}\text { Recuperabilidad (Rv) Mide la posibilidad de recuperar (total o } \\
\text { parcialmente) las condiciones de calidad ambiental iniciales como } \\
\text { consecuencia de la aplicación de medidas correctoras }\end{array}$} & 1 & Puede ser total e inmediata \\
\hline & 2 & Puede ser total a mediano plazo \\
\hline & 4 & Parcial \\
\hline \multirow{3}{*}{$\begin{array}{c}\text { Certidumbre }(\mathrm{Ce}) \text { Es el grado de seguridad con el que se espera } \\
\text { que se produzca el efecto }\end{array}$} & 1 & improbable \\
\hline & 2 & Probable \\
\hline & 3 & Cierto \\
\hline \multirow{4}{*}{$\begin{array}{l}\text { Importancia (I) Con la siguiente fórmula se mide la importancia del } \\
\text { impacto ambiental: } \quad I=+(\ln +\mathrm{Ex}+\mathrm{Mo}+\mathrm{Pe}+\mathrm{Rv}+\mathrm{Ce})\end{array}$} & $1<25$ & Irrelevante \\
\hline & $25<1<50$ & Moderado \\
\hline & $50<1<75$ & Severo \\
\hline & $\mid>75$ & Crítico \\
\hline
\end{tabular}

Fuente: elaboración propia con base en Vicente Conesa Fernández-Vítora (2010)

En la tabla 9 se presenta la matriz de Leopold que se obtuvo mediante la utilización el método Delphi.

TABLA 9

Matriz de Leopold (valores obtenidos utilizando el método Delphi)

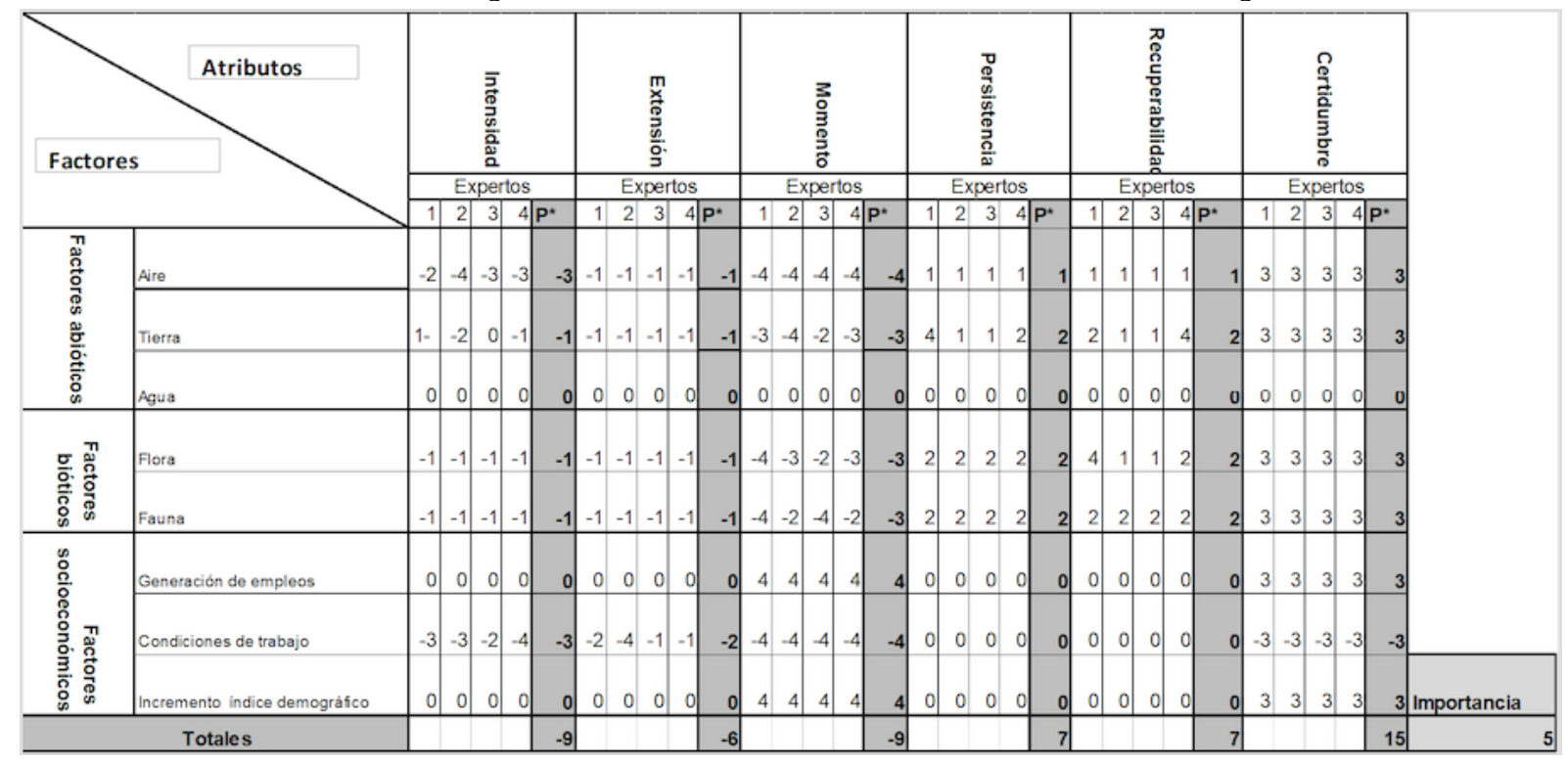

Fuente: elaboración propia

Cuatro expertos en plantas de beneficio de carbón mineral fueron seleccionados, para que con base en sus experiencias proporcionaron un valor para cada atributo en los tres factores que son bióticos, abióticos y socioeconómicos; se sumaron los cuatro valores proporcionados por los expertos y se obtuvo un promedio de cada atributo en los tres factores. 
La importancia del impacto surge de la siguiente fórmula (Conesa Fernández-Vítora, 2010).

$\mathrm{I}= \pm(\mathrm{In}+\mathrm{Ex}+\mathrm{Mo}+\mathrm{Pe}+\mathrm{Rv}+\mathrm{Ce})$

$\mathrm{Al}$ sustituir los valores que se obtuvieron en la matriz de Leopold, el resultado obtenido es el siguiente:

$\mathrm{I}=5$

Al considerar la información sobre la importancia del impacto ambiental que se presenta en la tabla 8 de los valores de los atributos, se puede concluir que este impacto es irrelevante ya que es menor a 25; sin embargo, uno de los expertos hizo mención a que sí hay un impacto visual.

\section{Conclusiones}

Las figuras 2 y 3 proporcionan información muy relevante, ya que se puede establecer una relación entre crecimiento poblacional en el municipio de San Javier, Sonora y la explotación de carbón mineral en esta región. La figura 2 indica que en los censos de 1980, 1990 y 2000, la población en este municipio iba decreciendo; sin embargo, en el censo del año 2010, la población tuvo una recuperación importante. Esta recuperación coincide con el incremento de la explotación de carbón mineral que se observa en la figura 3 en el período 2006-2014.

En lo que se refiere al desarrollo sostenible, cuidado del medio ambiente y sistema de producción más limpia - temas tratados en esta investigación - las conclusiones son las siguientes:

- Para que una empresa minera contribuya al desarrollo sostenible, debe considerar una mejor planeación estratégica y utilizar tecnologías más limpias. Según los resultados obtenidos en las encuestas, ninguna de las 5 plantas encuestadas tiene en sus instalaciones tecnologías limpias; por lo tanto, las plantas de beneficio de carbón mineral ubicadas en la zona centro del estado de Sonora no contribuyen al desarrollo sostenible.

- Para que una industria contribuya al cuidado del medio ambiente debe cumplir con las regulaciones actuales, y en el caso de las plantas encuestadas estas no cumplen algunos artículos de la Ley general de equilibrio ecológico y protección al ambiente (LGEEPA), de la Ley general para la prevención y gestión integral de los residuos (LGPGIR); tampoco cumplen las Normas Oficiales Mexicanas. Por lo tanto, se puede concluir que el cuidado del medio ambiente no es prioritario para los propietarios de las plantas de beneficio de carbón mineral ubicadas en San Javier, Sonora.

- En el tema de sistemas de producción más limpia, a partir de los resultados que se obtuvieron en los instrumentos aplicados, se puede concluir que ninguna empresa encuestada tiene en sus procesos sistemas de producción más limpia, ya que en todas se generan grandes cantidades de emisiones.

- Debido a que las plantas de beneficio de carbón mineral ubicadas en San Javier, Sonora, no tienen en la operación de sus instalaciones tecnologías limpias y no respetan algunas Leyes y Normas Oficiales Mexicanas, no contribuyen al desarrollo sostenible.

Como conclusión general, esta investigación proporciona información suficiente para garantizar que el problema planteado se puede solucionar mediante el Diseño de un modelo sostenible de producción más limpia en las operaciones de las plantas de beneficio de carbón mineral en el municipio de San Javier, Sonora.

\section{Referencias}

Conesa Fernández-Vítora, V. (2010). Guía metodológica para la evaluación del impacto ambiental. Madrid: Ediciones Mundi-Prensa.

Coria, I. D. (2008). El estudio de impacto ambiental: características y metodologías. Invenio, 11(20), 125-135. Recuperado de http://www.redalyc.org/articulo.oa?id=87702010 
Eggert, R. G. (2000). Sustainable Development and the Mineral Industry. En James M. Otto \& John Cordes, eds. Sustainable Development and the Future of Mineral Investment, Chapter 2, 2,1-2,15. Paris, France: United Nations Environment Programme, UNEP. Recuperado de http://apps.unep.org/redirect.php?file=/publications/pmtdocuments/Sustainable\%20Development\%20a nd\%20the\%20Future\%20of\%20Mineral\%20Investment-20001552.pdf

Espinosa-Murga, C. (2007). Cleaner Production Excellence Model. Geneva: Sustainable Business Associates, SBA. Recuperado de http://www.sba-int.ch/spec/sba/download/Publications\%20principales/CPExcellenceModel. $\mathrm{pdf}$

Flores-Galicia, E. (1988). Geología y reservas de los yacimientos de carbón en la república mexicana. En Guillermo P. Salas-Guerra. Geología económica de México, 175-217. Ciudad de México: Fondo de Cultura Económica, FCE.

Grinnell, R. M. \& Unrau, Y. (1977). Social Work Research \& Evaluation: Quantitative and Qualitative Approaches. Ithaca, New York: E. E. Peacock Publishers.

Grinnell, R. M., Williams, M., \& Unrau, Y. (2009). Research Methods for BSW Students. Kalamazoo, Michigan: Pair Bond Publications.

Hernández-Sampieri, R., Fernández-Collado, C., \& Baptista-Lucio, P. (2010). Metodología de la investigación. México: McGraw-Hill. Recuperado de https://www.esup.edu.pe/descargas/dep_investigacion/Metodologia\%20de\%20 la\%20investigaci\%C3\%B3n\%205ta\%20Edici\%C3\%B3n.pdf

Hilson, G. \& Murck, B. (2000). Sustainable Development in the Mining Industry: Clarifying the Corporate Perspective. Resources Policy, 26(4), 227-238. Recuperado de http://isiarticles.com/bundles/Article/pre/pdf/2 9041.pdf

Jackson, T. (1993). Clean Production Strategies: Developing Preventive Environmental Management in the Industrial Economy. Boca Ratón, Florida: Lewis Publishers.

Kayo, E. K. \& Securato, J. R. (1997). Método Delphi: Fundamentos, críticas e vieses. Cadernos de Pesquisa em Administração, Universidade de São Paulo, São Paulo, 1(4), 51-61.

Labonne, B. (1999). The Mining Industry and the Community; Joining Forces for Sustainable Social Development. Natural Resources Forum, 23(4), 315-322.

Leopold, L. B., Clarke, F. E., Hanshaw, B., B. \& Balsley, J. B. (1971). A Procedure for Evaluating Environmental Impact. Geological Survey Circular 645. Washington: United States Department of the Interior. Recuperado de https:/ /pubs.usgs.gov/circ/1971/0645/report.pdf

Martínez, M. (mayo de 2005). Gestiopolis.com. Recuperado de http://www.inteligenciacolectiva.org/principal_pro yectos_metodologia_delphi

Mayntz, R., Holm, K., \& Hübner, P. (1988). Introducción a los métodos de la sociología empirica. Madrid: Alianza Universidad.

Organización de las Naciones Unidas, ONU (1992). Declaración de Río sobre el Medio Ambiente y el Desarrollo. Conferencia de las Naciones Unidas sobre el Medio Ambiente en Río de Janeiro, 3-14 de junio de 1992. Recuperado de http://www.un.org/spanish/esa/sustdev/documents/declaracionrio.htm

Organización de las Naciones Unidas, ONU (2010). Resolución A/RES/64/212. Ciencia y tecnología para el desarrollo, aprobada por la Asamblea General el 21 de diciembre de 2009. New York: Asamblea General. Recuperado de http://www.un.org/en/ga/search/view_doc.asp?symbol=A/RES/64/212\&Lang=S

Secretaría de Economía del Estado de Sonora (2014). La minería en Sonora 2006-2012. Hermosillo, Sonora: Secretaría de Economía. Recuperado de http://www.2006-2012.economia.gob.mx/files/transparencia/informe_APF/de legaciones/sonora.pdf

Selltiz, C., Wrightsman, L., \& Cook, S. W. (1980). Métodos de la investigación en las relaciones sociales. Madrid: RIALP.

\section{Notas}

* Artículo de investigación científica y tecnológica. 
Licencia Creative Commons CC BY 4.0

Para citar este artículo: Duarte-Verdugo, Luis Enrique; Robles-Ibarra, Juan Carlos; Pico-González, Beatriz \& Rosano-Ortega, Genoveva (2017). Estudio empírico en plantas de beneficio de carbón mineral localizadas en San Javier, Sonora, México, para diseñar un sistema sostenible de producción más limpia. Cuadernos de Contabilidad, 18(45), 40-53. https://doi.org/10.11144/Javeriana.cc18-45.eepb 